\title{
Editorial
}

Interational Archives of
Allergy
Immund

\section{How Low Should We Go?}

\author{
Julia Upton Thomas Eiwegger \\ Division of Immunology and Allergy, Food Allergy and Anaphylaxis Program, Department of Pediatrics, \\ Hospital for Sick Children, University of Toronto, Toronto, Ont., Canada
}

Milk allergy is a common phenomenon with a prevalence of around 2\% in 2-year-old children. Avoidance itself is not sufficient to prevent allergic reactions as suggested by one study reporting an incidence of $40 \%$ allergic reactions during a 12 -month period. Thirty-seven percent of reactions were moderate to severe [1]. Therefore feasible treatment options for milk allergy are needed. Milk oral immunotherapy (OIT) has been investigated for treatment of milk allergy [2]. Many OIT trials targeted serving-size amounts of food such as approximately 5$16 \mathrm{~g}$ in milk, $2 \mathrm{~g}$ in egg and $0.8-7 \mathrm{~g}$ in peanut OIT trials. Initial enthusiasm over OIT to milk was tempered by allergic reactions (19\% with anaphylactic reaction at least once) and by limited long-term responders (31\%) [3].

The OIT method of the recent long-term follow-up study of milk used $500 \mathrm{mg}$ milk protein and had a median maximum tolerated dose after OIT of 1,400 mg [3]. Previous studies in peanut have shown that low-dose OIT (LOIT) may have promise as a treatment. Recently, an abstract has attracted attention at the AAAAI [4]. In this study, yet to be reported unblinded, it appeared that there was little difference in clinical and immunological outcomes in young children randomized to low-dose (300 $\mathrm{mg})$ or high-dose $(3,000 \mathrm{mg})$ OIT to peanut. Previous to that study, the same center initially targeted a maintenance dose of $300 \mathrm{mg}$ of peanut protein and $93 \%$ who completed the protocol passed a challenge of 3,900 $\mathrm{mg}$ peanut protein without symptoms [5]. Blumchen et al. [6] targeted $500 \mathrm{mg}$ of whole crushed peanuts, corresponding to $125 \mathrm{mg}$ peanut protein, and found that some participants tolerated $4 \mathrm{~g}$ of crushed peanuts. Therefore, it is documented that participants could tolerate more than $10 \times$ the dose used for maintenance OIT. Other methodologies of immunotherapy which target lower maintenance doses than many OIT protocols include sublingual immunotherapy to milk and epicutaneous immunotherapy [reviewed in 2] (fig. 1).

If ingestion of a very low amount of food can elicit a clinical reaction, then perhaps very low doses can also be immune modifying. A review of published trials found that the minimum eliciting dose was $3.3 \mathrm{mg}$ for milk [7]. This amount would correspond to about $0.009 \mathrm{ml}$ or less than $1 / 4$ of a drop of milk.

In a recent edition of the International Archives of Allergy and Immunology, Yanagida et al. [8] explore the use of an LOIT protocol to milk in a severely milk-allergic population. The maintenance dose target was only $3 \mathrm{ml}$ of milk or just over $100 \mathrm{mg}$ of milk protein. This dose is less than $2 \%$ of what would traditionally be considered a serving of milk. They used an initial 5-day in-hospital dose escalation protocol with ongoing daily doses and any required dose escalation was at home. Of the $12 \mathrm{pa}-$ tients on treatment, $9 / 12$ could drink $3 \mathrm{ml}$ of milk daily without reactions and $4 / 12$ could tolerate $25 \mathrm{ml}$, which

\section{KARGER 125}

(c) 2016 S. Karger AG, Base

$1018-2438 / 16 / 1683-0147 \$ 39.50 / 0$
Correspondence to: Dr. Thomas Eiwegger

Division of Immunology and Allergy, Food Allergy and Anaphylaxis Program, Department of Pediatrics, Hospital for Sick Children, University of Toronto 555 University Avenue, Toronto, ON M5G 1X8 (Canada)

E-Mail Thomas.Eiwegger@ sickkids.ca 


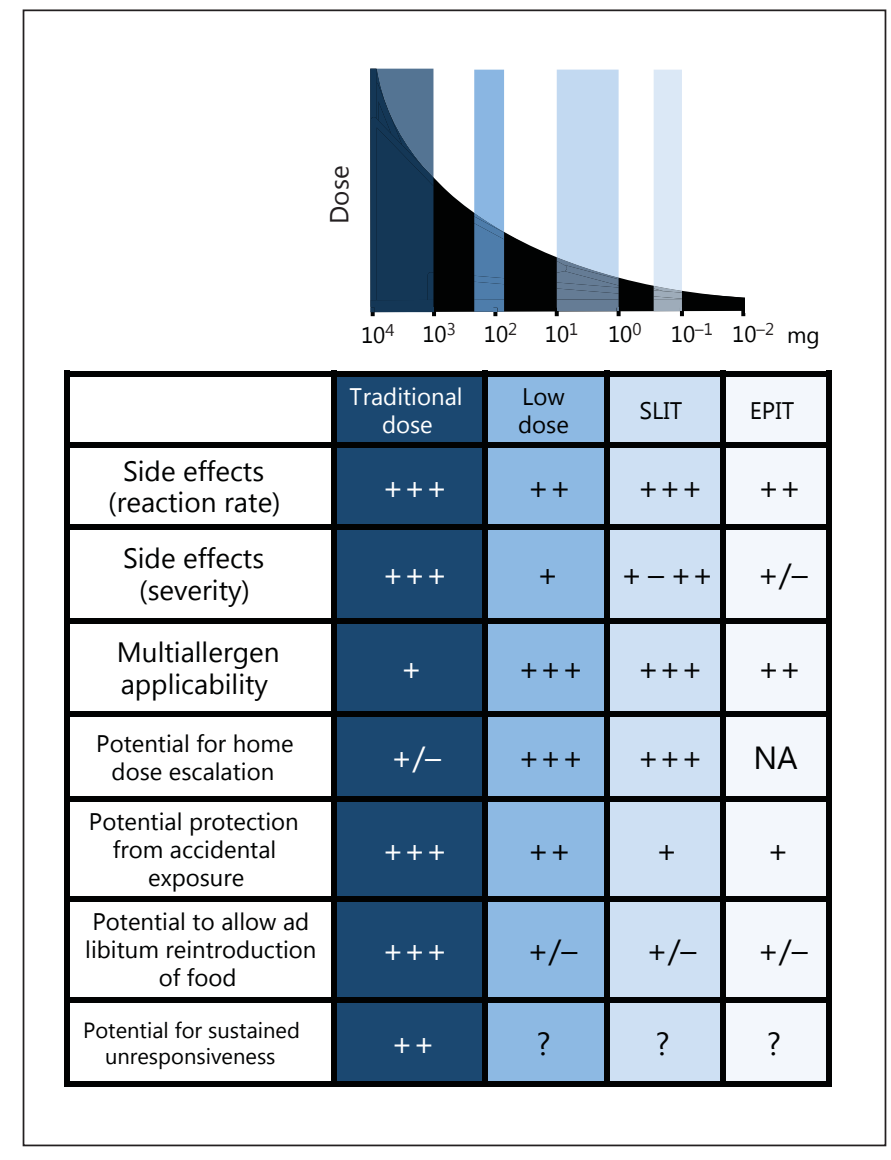

Fig. 1. Methods of food allergen immunotherapy. EPIT = Epicutaneous immunotherapy; $\mathrm{NA}=$ no data available.

was more than 8 -fold the amount of the OIT maintenance target. Along with these clinical changes a decrease in specific IgE to casein in the OIT group was observed.

Importantly, this study included high-risk patients as defined both in terms of clinical reactions at a low dose at the initial food challenge and also more than $90 \%$ had a history of anaphylaxis. The applicability of their protocol is intriguing. This is reflected by the fact that despite the severity of the symptoms of the patients, they had no dropouts in the treatment arm. While initial dosing was performed in hospital, ongoing up-dosing was performed at home. It would appear that a low-dose protocol like theirs may require fewer resources than traditional OIT which is mainly restricted to academic settings and therefore may be more likely to be applicable in a community setting. A significant reduction of food allergy-induced clinical burden will likely require therapeutic and prevention strategies which include multifood desensitization strategies. LOIT may make multifood desensitization protocols more feasible.

This study is also informative due to nonresponders. The authors found that $3 / 12$ could not drink $3 \mathrm{ml}$ of milk without symptoms even after a year of treatment. This finding further contributes to the growing observation that there is a group of patients who have great difficulty with the OIT process. Even at this very low-dose target their nondesensitized rate is in keeping with the usual 15-20\% rate of patients who cannot complete OIT seen across OIT studies [2]. It is vital to find new markers to define nonresponders who are at risk.

In addition to the limitations stated in the article, a few other points can be discussed. It was not assessed whether baked milk is tolerated. Baked milk-tolerant patients may not warrant this LOIT approach because of their relatively good prognosis [9]. Another issue is the relative short period of avoidance after the treatment. Two weeks of avoidance are maybe too short to assess true tolerance development. Nevertheless $1 / 12$ of the originally desensitized patients reacted to $3 \mathrm{ml}$ underlining the importance of compliance in OIT. A longer duration of milk avoidance may increase this rate of failures further. Additionally, the oral challenge material was baked for $1.5 \mathrm{~min}$ in the microwave, which may have reduced its allergenicity. However, the 25-ml follow-up challenge was performed with regular milk.

It is important to consider the expectations and goals of LOIT. The amount used in this study should provide some accidental exposure protection. In the largest study of cross-contamination amounts in labeled and unlabeled food products, the amount of milk contamination ranged from 0.3 to $7.3 \mathrm{mg}$ per serving [10]; therefore, the amount of milk used in this study should provide some clinical protection. However, it would not be expected to allow ad libitum ingestion of milk and thus would require ongoing avoidance and epinephrine autoinjector availability.

There is need for caution before implementing this low-dose strategy. Although the rate of moderate and severe reactions at home were low, even with such a lowdose target patients still had reactions. With a longerterm follow-up it would be important to know whether these patients on LOIT can continue to increase their thresholds, whether they have better compliance, and whether they are at lower risk from their daily doses than traditional OIT. Most importantly, it would be important to know how low is too low to have a clinically meaningful effect.

As recently reviewed, the available evidence is pointing towards only a few patients achieving sustained unre- 
sponsiveness with the current methodologies, especially for milk [2]. Therefore, long-term treatments may be required and efficacy, compliance and safety will be key considerations for future treatments. LOIT may have a role in OIT (fig. 1) because of the feasible administration in patients that are not eligible for high-dose OIT and potential cost savings in resources and immunotherapy materials. Since the dose tolerated after OIT can be $10 \times$ higher than the treatment dosing it may give a comfortable buffer for accidental exposures.

\section{References}

1 Boyano-Martínez T, García-Ara C, Pedrosa M, Díaz-Pena JM, Quirce S: Accidental allergic reactions in children allergic to cow's milk proteins. J Allergy Clin Immunol 2009;123: 883-888.

2 Sato S, Yanagida N, Ogura K, Imai T, Utsunomiya $\mathrm{T}$, Iikura $\mathrm{K}$, et al: Clinical studies in oral allergen-specific immunotherapy: differences among allergens. Int Arch Allergy Immunol 2014;164:1-9.

3 Keet CA, Seopaul S, Knorr S, Narisety S, Skripak J, Wood RA: Long-term follow-up of oral immunotherapy for cow's milk allergy. J Allergy Clin Immunol 2013;132:737-739.e6.
4 Vickery BP, Steele PH, Kamilaris JS, Burk C, Kamilaris N, Kulis MD, et al: Low-dose oral immunotherapy as an early intervention strategy for peanut allergy. J Allergy Clin Immunol 2013;131:AB130.

5 Jones SM, Pons L, Roberts JL, Scurlock AM, Perry TT, Kulis M, et al: Clinical efficacy and immune regulation with peanut oral immunotherapy. J Allergy Clin Immunol 2009;124: 292-300, 300.e1-e97.

6 Blumchen K, Ulbricht H, Staden U, Dobberstein K, Beschorner J, de Oliveira LCL, et al: Oral peanut immunotherapy in children with peanut anaphylaxis. J Allergy Clin Immunol 2010;126:83-91.e1.

7 Zhu J, Pouillot R, Kwegyir-Afful EK, Luccioli S, Gendel SM: A retrospective analysis of allergic reaction severities and minimal eliciting doses for peanut, milk, egg, and soy oral food challenges. Food Chem Toxicol 2015;80:92-100.
8 Yanagida N, Sato S, Asaumi T, Okada Y, Ogura K, Ebisawa M: Single-center case-control study of low-dose induction oral immunotherapy with cow's milk. Int Arch Allergy Immunol 2015;168:131-137.

9 Leonard SA, Caubet J-C, Kim JS, Groetch M, Nowak-Węgrzyn A: Baked milk- and eggcontaining diet in the management of milk and egg allergy. J Allergy Clin Immunol Pract 2015;3:13-23; quiz 24.

10 Ford LS, Taylor SL, Pacenza R, Niemann LM, Lambrecht DM, Sicherer SH: Food allergen advisory labeling and product contamination with egg, milk, and peanut. J Allergy Clin Immunol 2010;126:384-385. 